\title{
Manifestaciones orales en pacientes VIH/SIDA asociadas a tratamiento antirretroviral y el estado inmunológico en dos fundaciones de la ciudad de Cartagena
}

\section{Oral manifestations in HIV/AIDS patients associated with antiretroviral treatment and immune status in two foundations of Cartagena city}

\author{
Jiménez Malagón MC*, Harris Ricardo J**, Palomino Rossi W**, \\ Díaz Caballero AJ***, Puello del Río E****
}

\begin{abstract}
RESUMEN
Objetivo: El objetivo de este estudio fue determinar la asociación entre las manifestaciones orales de pacientes $\mathrm{VIH} / \mathrm{SIDA}$ con la terapia antirretroviral y el estado inmunológico.

Metodología: Estudio de tipo transversal, en una población de 166 pacientes pertenecientes a dos fundaciones de la ciudad de Cartagena, la información se recolectó a través de un examen estomatológico intraoral teniendo en cuenta los criterios para el diagnóstico clínico de las manifestaciones orales asociadas a VIH establecidos por el Centro Colaborador de la OMS sobre las manifestaciones orales del virus de inmunodeficiencia humana, los datos como niveles de carga viral, CD4 y la terapia antirretroviral se obtuvieron de la historia clínica médica. Resultados: La prevalencia de manifestaciones orales asociadas a VIH fue del 59,5\%, la candidiasis fue la manifestación oral más frecuente, con una prevalencia de $35,5 \%$, los pacientes tratados con monoterapia presentaron menos manifestaciones orales que aquellos tratados con biterapia con valores estadísticamente significativo, OR: 0,20, IC: 0,00-0,96 ( $\mathrm{p}<0,02)$, la candidiasis pseudomembranosa como manifestación oral asociada a $\mathrm{VIH}$ tiene menos probabilidades de presentarse cuando se tiene niveles de CD4 superiores a 500 cel./mm $\mathrm{mm}^{3}$ mostrando valores estadísticamente significativos, con un OR: 0,297, IC: 0,103-0,852 ( $<<0,03$ ). Conclusión: Los pacientes tratados con monoterapia como terapéutica farmacológica antirretroviral presentaron menos manifestaciones orales que aquellos tratados con biterapia, con respecto a la candidiasis el subtipo pseudomembranosa tiene menos probabilidades de presentarse cuando se tiene niveles de CD4 superiores a 500 cel. $/ \mathrm{mm}^{3}$.
\end{abstract}

Palabras clave: Carga viral, antígenos CD4, infecciones por VIH (Decs Bireme).

\section{SUMMARY}

Objective: The aim of this study was to determine the association between oral manifestations of AIDS patients with antiretroviral therapy and their immune status.

* Odontólogo. Universidad de Cartagena. Especialista en Estomatología y Cirugía Oral Universidad de Cartagena. Profesor titular Facultad de Odontología Universidad de Cartagena.

** Odontólogo. Universidad del Sinú Seccional Cartagena. Especialista en Estomatología y Cirugía Oral, Universidad de Cartagena.

*** Odontólogo. Universidad de Cartagena. Especialista en Periodoncia Universidad Javeriana. Profesor titular Universidad de Cartagena. Director Grupo de investigaciones GITOUC.

**** Odontólogo Universidad de Cartagena. Especialista en Cirugía Oral Universidad de São Pablo, Brasil. Profesor titular Facultad de Odontología Universidad de Cartagena. 
Methodology: a cross-sectional study was done in a population of 166 patients from two Cartagena's city foundations, the information was collected through an intraoral stomatological examination considering the standard for the clinical diagnosis of oral manifestations associated with AIDS established by the Collaborating Centre WHO about oral manifestations of human immunodeficiency virus, information such as viral load, CD4 and antiretroviral therapy were obtained from the medical history.

Results: The prevalence of oral manifestations associated with AIDS was 59.5\%, candidiasis was the most common oral manifestation with a prevalence of $35.5 \%$, the patients treated with oral monotherapy had fewer events than those treated with combination therapy with statistically significant values, OR: $0.20, \mathrm{Cl} 0.00-0.96$ $(\mathrm{p}<0.02)$, pseudomembranous candidiasis as oral manifestations associated with AIDS has less probabilities to occur when patients have CD4 levels above $500 \mathrm{cel} . / \mathrm{mm}^{3}$ showing values statistically significant with a OR: 0.297, CI: 0103-0852 ( $<<0.03)$.

Conclusions: Patients treated with monotherapy as antiretroviral drug therapy presented less oral manifestations than patients treated with dual therapy, about candidiasis, the subtype pseudomembranous candidiasis has less probabilities to occur when you have CD4 levels above $500 \mathrm{cel} . / \mathrm{mm}^{3}$.

Key words: Viral load, antigens CD4, HIV Infections (Mesh Database).

Fecha de recepción: 26 de abril de 2011.

Aceptado para publicación: 10 de mayo de 2011.

Jiménez Malagón MC, Harris Ricardo J, Palomino Rossi W, Díaz Caballero AJ, Puello del Río E. Manifestaciones orales en pacientes VIH/SIDA asociadas a tratamiento antirretroviral y el estado inmunológico en dos fundaciones de la ciudad de Cartagena. Au. Odontoestomatol 2012; 28 (4): 181-189.

\section{INTRODUCCIÓN}

La Organización Mundial de la Salud (OMS) y el Programa Conjunto de las Naciones Unidas sobre el $\mathrm{VIH} / \mathrm{SIDA}$ calcularon que para finales del año 2008, las nuevas cifras de infecciones por el Virus de la Inmunodeficiencia Humana fueron de 2,7 millones y estimaron que para ese año el número de defunciones en el mundo por enfermedades relacionadas con el Sida fue de 2 millones; la mayoría de ellos (70\%) habitaban en África y seguidos por América Latina y el Caribe $(1,2)$.

El comportamiento de la epidemia de VIH-SIDA en Colombia presenta una transición demográfica progresiva del predominio en varones homosexuales al predominio en población heterosexual femenina y masculina, pasando de una relación hombre-mujer de 12:1 a 5:1; esta progresión es más evidente en algunas regiones como la costa atlántica donde la relación llega a ser de 3:1. El total de casos notificados a nivel nacional desde 1983 hasta el 31 de diciembre de 2005 fue de 46.809 casos (3).
La infección por el VIH provoca una depleción en los linfocitos CD4, esto tiene como consecuencia una alteración y disminución de la inmunidad celular que se acompaña de un incremento de las infecciones oportunistas (4), diversos autores como Patton et al, Estrada J y cols., Coogan M et al, destacan la Candidiasis como una de las primeras manifestaciones por la infección en cavidad oral, siendo esta la más frecuente asociada a $\mathrm{VIH}(5-7)$.

La terapia con antirretrovirales modificó la historia natural de la infección e indujo a un cambio en el curso y pronóstico de la enfermedad, anteriormente la supervivencia no era superior al $30-50 \%$ a los 2 años y menor del $10-20 \%$ a los 4 años. El recuento de los linfocitos T CD4 y el nivel de ARN plasmático del VIH-1 determinado por técnicas de carga viral ofrecen una primera aproximación como predictores de la progresión de la infección VIH/SIDA $(8,9)$. El tratamiento reduce la carga viral del plasma y ataca el virus en diversas etapas de su ciclo de la réplica, esta reducción permite la reconstitución del sistema inmune que mejorará la resolución de infecciones y la reduc- 
ción de riesgos para acontecimientos secundarios; pero no está claro si las manifestaciones orales solo se deben a la inmunosupresión o a los efectos secundarios de los antirretrovirales, entre los fármacos antirretrovirales se incluyen los inhibidores nucleósido de transcriptasa reversa (INTR), los inhibidores no nucleósido de transcriptasa reversa (INNTR), inhibidores de proteasa (IP) y los inhibidores de fusión (10,11), los factores que predisponen la expresión de lesiones orales incluyen conteos de CD4 menores de 200 células $/ \mathrm{mm}^{3}$, una carga viral mayor de 3.000 copias/ml (12).

Tappuni AR y Fleming GJ, en el año 2001, realizaron un estudio de tipo transversal, en Inglaterra donde determinaron el efecto de la terapia antirretroviral en la prevalencia de manifestaciones orales en pacientes $\mathrm{VIH}$, demostraron que las manifestaciones orales se disminuían en pacientes con terapia dual y terapia triple en comparación con los que recibían monoterapia; también encontraron un incremento de las manifestaciones orales en pacientes con conteo de CD4 menores a 200 células $/ \mathrm{mm}^{3}$ y carga viral mayores a 3.000 copias $/ \mathrm{ml}$ (13). Existen pocos estudios que caractericen la asociación de las manifestaciones orales con la terapia antirretroviral, los niveles de CD4 y carga viral; el objetivo del presente estudio fue evaluar el comportamiento de esa asociación.

\section{MÉTODO}

Se realizó un estudio transversal en el cual se determinó la asociación entre las manifestaciones orales de pacientes $\mathrm{VIH} / \mathrm{SIDA}$ con la terapia antirretroviral y el estado inmunológico. La población correspondió a 166 pacientes, usuarios inscritos en el programa de VIH//SIDA de dos Fundaciones en la ciudad de Cartagena de Indias y solo 141 pacientes cumplieron con los criterios de inclusión.

En el estudio se incluyeron pacientes independientes de edad, sexo y raza, que estuvieran bajo tratamiento farmacológico con antirretrovirales sin interrupción, con seguimiento de linfocitos CD4 y carga viral menor a 30 días del examen clínico intraoral, que firmaron previamente el consentimiento informado, los aspectos éticos se adecuaron a las recomendaciones para investigación biomédica de Helsinki de la Asociación Médica Mundial y de las normas científico téc- nicas y administrativas para la investigación en salud resolución No. 008430 de 1.993 emanada del Ministerio de Salud de Colombia en su título II Capítulo I sobre los aspectos éticos de la investigación en humanos Artículos 5 al 11. Este es un estudio de riesgo mínimo como lo estipula la resolución No. 008430 de 1993 y la ley 84 de 1989. No se incluyeron las patologías orales o sistémicas padecidas por los pacientes antes de adquirir la infección por el VIH y los pacientes que no estaban en terapia antirretroviral.

Se estudiaron variables como las características de las patologías orales, la carga viral, valores absolutos de linfocitos T CD4 y la terapia antirretroviral, además de los aspectos sociodemográficos de importancia.

Se realizó anamnesis y examen clínico intraoral teniendo en cuenta los criterios para el diagnóstico clínico de las manifestaciones orales asociadas a $\mathrm{VIH}$ establecidos por el Centro de intercambio de criterios sobre problemas bucales relacionados con la infección por el VIH (CE) y el Centro Colaborador de la OMS sobre las manifestaciones orales del virus de inmunodeficiencia humana (14), los datos correspondientes a niveles de CD4, carga viral y terapia antirretroviral fueron obtenidos de la historia clínica médica, dichos exámenes de laboratorio no debían pasar un tiempo superior a 30 días del examen clínico intraoral.

Se realizó la prueba piloto con el $10 \%$ del número del total de la población, en ella participaron pacientes con VIH que asistían a la consulta de la facultad de odontología, para estandarizar los criterios diagnósticos en los examinadores, se realizó un análisis interexaminador cada paciente fue examinado por los dos investigadores independientemente para controlar las variaciones entre los examinadores. Para analizar esa información se aplicó el índice KAPPA, diseñándose una tabla de clasificación cruzada cuyo resultado arrojó un valor de 0,84 tomando dicho resultado como calibración positiva, puesto que superó el valor de 0,80 .

\section{ANÁLISIS DE LA INFORMACIÓN}

El análisis estadístico de la información se procesó en paquete estadístico Stata 9.2 Se calculó a través 
de indicadores de frecuencia como porcentajes y prevalencia para saber cual o cuales patologías fueron las más frecuentes, que terapia antirretroviral estaban recibiendo y los niveles de carga viral y de CD4. Para analizar la relación entre las variables patologías orales y terapia antirretroviral (análisis bivariado) se calcularon estimadores de asociación (Odds Ratio). Considerando resultados significativos a un valor de p menor o igual a 0,05.

\section{RESULTADOS}

Del total de pacientes atendidos en las dos fundaciones de la ciudad de Cartagena se excluyeron 16 pacientes por no presentar estudios paraclínicos con vigencia a 30 días y 9 pacientes por no estar bajo terapia farmacológica, para un total de 141 pacientes evaluados, de estos el $66,7 \%$ fueron hombres, la edad promedio fue de 36,1 años con una desviación estándar (D.E) de 11,7 años, el 53,9\% de los pacientes presentaron niveles educativos hasta la secundaria, mientras que el $5,0 \%$ de la población no presentó ningún tipo de estudio y el $84,4 \%$ era de procedencia urbana. En cuanto al mecanismo de transmisión del virus el método más frecuente fue el sexual con un $96 \%$, el tiempo promedio de diagnóstico de la infección con el virus fue de 4,2 años con una (D.E) de 3,31 años (Tabla 1).

Entre las evaluaciones se detectaron 18 patologías orales en el $88.6 \%(n=141)$ de los pacientes, en di-

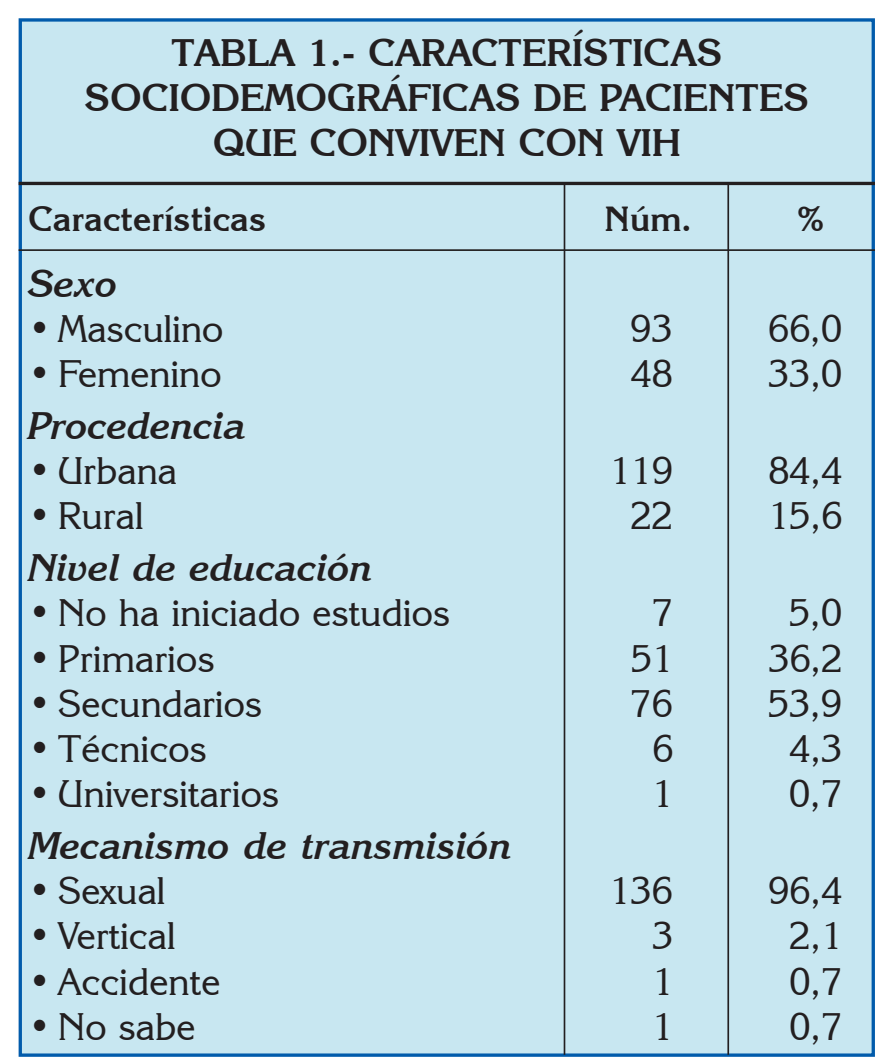

ferentes localizaciones de la boca, de estos la prevalencia de manifestaciones orales asociadas a $\mathrm{VIH}$ fue del $59,5 \%$ del total de pacientes evaluados durante este estudio. Las patologías de mayor prevalencia fueron: candidiasis pseudomembranosa $31,3 \%$, gingivitis marginal lineal $10 \%$, candidiasis eritematosa 4,2\%, xerostomía 3,3\% (Tabla 2).

TABLA 2.- RELACIÓN MANIFESTACIONES ORALES, NIVELES DE CD4 Y CARGA VIRAL

\begin{tabular}{|c|c|c|c|c|c|c|c|}
\hline \multirow[b]{2}{*}{ Manifestaciones } & \multirow[b]{2}{*}{$\begin{array}{l}\text { Número } \\
\mathrm{N}=82\end{array}$} & \multirow[b]{2}{*}{$\%$} & \multicolumn{3}{|c|}{$\begin{array}{l}\text { Niveles de CD4 } \\
\mathrm{N}^{\circ} \text { de casos }\end{array}$} & \multicolumn{2}{|c|}{$\begin{array}{c}\text { Carga viral } \\
\mathrm{N}^{\circ} \text { de casos }\end{array}$} \\
\hline & & & $\begin{array}{c}>500 \\
\text { células/ml }\end{array}$ & \begin{tabular}{|l}
$200-499$ \\
células/ml
\end{tabular} & $\begin{array}{c}<200 \\
\text { células/ml } \\
\end{array}$ & $\begin{array}{c}<50.000 \\
\text { copias/ml }\end{array}$ & $\begin{array}{l}>50.000 \\
\text { copias } / \mathrm{ml}\end{array}$ \\
\hline Estomatitis aftosa recurrente & 2 & 1,3 & 0 & 1 & 1 & 1 & 1 \\
\hline Candidiasis eritematosa & 6 & 4,2 & 2 & 2 & 2 & 4 & 2 \\
\hline Candidiasis pseudomembranosa & 47 & 31,3 & 6 & 19 & 22 & 30 & 17 \\
\hline Papiloma & 4 & 2,7 & 1 & 3 & 0 & 4 & 0 \\
\hline Gingivitis marginal & 15 & 10,0 & 4 & 8 & 3 & 8 & 7 \\
\hline Periodontitis del adulto & 3 & 2,0 & 0 & 1 & 2 & 2 & 1 \\
\hline Xerostomía & 5 & 3,3 & 1 & 0 & 4 & 5 & 0 \\
\hline
\end{tabular}


En cuanto a las manifestaciones orales la que se presentó con más frecuencia fue la candidiasis pseudomembranosa con un 31,3\%, manifestándose en 47 pacientes, de estos solo 22, presentaron valores de CD4 menores de $200 \mathrm{cell} / \mathrm{mm}^{3}$ y 30 pacientes con valores menores de 50.000 copias (Tabla 2).

Con respecto a la farmacoterapia de los 141 pacientes que recibieron tratamiento, el $56,7 \%$ recibió terapia conjugada con IP/INTR, seguido de un $26,2 \%$ con terapia INTR/INNTR, en menor proporción un $2,8 \%$ con IP. La manifestación oral más frecuente según la terapia IP/INTR (53 pacientes) fue Candidiasis pseudomembranosa con 29 pacientes. En relación con los pacientes que presentaron manifestaciones orales asociadas a VIH $(n=82)$ el $11,3 \%$ estaba bajo esquema antirretroviral monoterapia y el $88,7 \%$ con biterapia (Tablas 3 y 4).

El 46,1\% de los pacientes tenían valores de CD4 entre 200-499 cell/ $/ \mathrm{mm}^{3}$, de los 82 pacientes que mostraron manifestaciones orales asociadas 37 pacientes presentaron valores entre $200-499 \mathrm{cell} / \mathrm{mm}^{3}$, siendo la Candidiasis pseudomembranosa la patología más frecuente con 19 pacientes. El 73,8\% de los pacientes tenían carga viral menor a 50.000 copias de los 82 pacientes que presentaron manifestaciones asociadas 54 pacientes tenían cargas virales inferiores a 50.000 copias, presentándose la candidiasis pseudomembranosa como la patología más frecuente con 30 pacientes (Tablas 1-6).

\section{TABLA 3.- FARMACOTERAPIA, NIVELES DE CD4 Y CARGA VIRAL EN PACIENTES QUE CONVIVEN CON VIH}

\begin{tabular}{|l|c|c|}
\hline Variable & $\mathbf{n}=\mathbf{1 4 1}$ & $\%$ \\
\hline Farmacoterapia actual & & \\
- IP/INTR & 80 & 56,7 \\
- INTR/INNTR & 37 & 26,2 \\
- INTR & 12 & 8,5 \\
- IP/INNTR & 8 & 5,7 \\
- IP & 4 & 2,8 \\
Niveles de CD4 & & \\
- >500 células/ml & 28 & 19,9 \\
- 200-499 cel./ml & 65 & 46,1 \\
- <200 células/ml & 48 & 34,0 \\
Niveles de carga viral & & \\
$<50.000$ copias/ml & 104 & 73,8 \\
$>50.000$ copias/ml & 37 & 26,2 \\
\hline
\end{tabular}

La candidiasis pseudomembranosa tiene menos probabilidades de presentarse cuando se tiene niveles de CD4 superiores a 500 cell. $/ \mathrm{mm}^{3}$ OR: 0,297, IC: 0,103-0,852 ( $<<0,03)$ (Tabla 5).

En relación a la terapia antirretroviral y el riesgo de presentar manifestaciones orales asociadas a $\mathrm{VIH}$, se encontró que recibir monoterapia tiene un efecto

\section{TABLA 4.- RELACIÓN MANIFESTACIONES ORALES Y TERAPIA ANTIRRETROVIRAL}

\begin{tabular}{|c|c|c|c|c|c|c|c|}
\hline \multirow[b]{3}{*}{ Manifestaciones } & \multirow[b]{3}{*}{$\begin{array}{c}\text { Número } \\
\mathrm{N}=82\end{array}$} & \multirow[b]{3}{*}{$\%$} & \multicolumn{5}{|c|}{$\begin{array}{c}\text { FARMACOLOGÍA } \\
\text { Número de casos }\end{array}$} \\
\hline & & & \multicolumn{2}{|c|}{ Monoterapia } & \multicolumn{3}{|c|}{ Biterapia } \\
\hline & & & INTR & IP & IP/INTR & INTP/INNTR & IP/INNTR \\
\hline Estomatitis aftosa recurrente & 2 & 1,3 & 0 & 0 & 2 & 1 & 0 \\
\hline Candidiasis eritematosa & 6 & 4,0 & 0 & 0 & 0 & 4 & 2 \\
\hline Candidiasis pseudomembranosa & 47 & 31,3 & 4 & 0 & 14 & 30 & 0 \\
\hline Papiloma & 4 & 2,7 & 0 & 0 & 0 & 4 & 0 \\
\hline Gingivitis marginal & 15 & 10,0 & 1 & 0 & 2 & 8 & 1 \\
\hline Periodontitis del adulto & 3 & 2,0 & 1 & 0 & 0 & 2 & 0 \\
\hline Xerostomía & 5 & 3,3 & 0 & 0 & 2 & 5 & 0 \\
\hline
\end{tabular}




\section{TABLA 5.- RELACIÓN DE NIVELES DE CD4 CON EL RIESGO DE PRESENTAR MANIFESTACIONES ORALES}

\begin{tabular}{|l|c|c|c|}
\hline Variable & OR & IC & P \\
\hline $\begin{array}{l}\text { Candidiasis } \\
\text { seudomembranosa }\end{array}$ & 0,297 & $0,103-0,852$ & 0,0329 \\
$\begin{array}{l}\text { Melanosis fisiologica } \\
\text { Xerostomía }\end{array}$ & 0,484 & $0,048-4,870$ & 0,538 \\
$\begin{array}{l}\text { Gingivitis marginal } \\
\text { asociada a placa }\end{array}$ & 0,484 & $0,048-4,870$ & 0,538 \\
$\begin{array}{l}\text { Candidiasis } \\
\text { eritematosa }\end{array}$ & 2,143 & $0,446-10,295$ & 0,341 \\
Lengua geográfica & 1,533 & $0,205-11,481$ & 0,677 \\
Melanosis por tabaco & 0,742 & $0,064-8,540$ & 0,811 \\
\hline
\end{tabular}

de menor riesgo de aparición de manifestaciones orales OR: 0,20, IC: 0,00-0,96 ( $<<0,02)$ (Tabla 6).

\section{DISCUSIÓN}

En el presente estudio la prevalencia de manifestaciones orales asociadas a VIH fue del $59,5 \%$, en la fundación número 1 fue del $59 \%$ y en la fundación número 2 fue del 51,1\%, mostrándose mayor diferencia con el estudio realizado por Ranganathan $\mathrm{K}$ et al, la prevalencia que se mostró fue del 86,6\% (15), mientras que otros estudios mostraron diferencias menores con el realizado por Laurenco AG et al, que representó el 33,2\% (16) y Tappuni AR et al, donde la prevalencia de manifestaciones orales asociadas a $\mathrm{VIH}$ fue del 30\%; (13), lo que puede explicar una relación entre las manifestaciones orales como potencial marcador de la infección por el (VIH).

TABLA 6.- RELACIÓN DE LA TERAPIA ANTIRRETROVIRAL COMO FACTOR DE RIESGO PARA MANIFESTACIONES ORALES ASOCIADAS A VIH

\begin{tabular}{|l|c|c|c|}
\hline Variable & OR & IC & P \\
\hline Monoterapia & 0,20 & $0,00-0,96$ & 0,02049 \\
\hline
\end{tabular}

Con relación a la frecuencia de la candidiasis como la manifestación oral más frecuente, en este estudio fue de 35,5\%, presentándose esta relación en otros estudios de diversos autores como Tappuni AR et al, que reportó el $30 \%$ (13), Bravo IM et al, el cual reportó prevalencia del $61 \%$ estadísticamente significativa $\mathrm{p}<0,001$ (17); Miziara ID et al, que evaluó la inmunosupresión en niños con VIH tratados con terapia antirretroviral fue del 45,9\% (18). Con respecto a los tipos de candidiasis que se presentaron, el subtipo pseudomembranosa en $86,7 \%$; mostrándose poca diferencia con el estudio de Ceballos A y cols, donde solo determinaron la prevalencia de lesiones orales por cándida en pacientes con sida sometidos a terapia antirretroviral altamente activa representando el $79,5 \%$ (19); lo que explica que la candidiasis es una de las manifestaciones orales frecuentemente asociada a la enfermedad relacionándose ampliamente con el grado de inmunosupresión.

Referente a la terapia farmacológica antirretroviral el $12 \%$ de los pacientes estaban siendo tratados con monoterapia y $88 \%$ con biterapia, Tappuni AR et al, demostraron que el $13 \%$ fueron tratados con monoterapia, $46 \%$ estaban con tratamiento dual y el $40 \%$ en terapia triple (13). En este estudio los pacientes tratados con monoterapia presentaron menos manifestaciones orales que aquellos tratados con biterapia con valores estadísticamente significativo, OR: 0,20, IC: 0,00-0,96 ( $<<0,02)$, difieren de Tappuni AR et al, que demostraron que los pacientes tratados con monoterapia tuvieron significativamente más lesiones orales que los sujetos de doble y triple terapia $\mathrm{p}<0,05$ (13), Hamza OJ et al, reportaron que los pacientes adultos con más de un fármaco antirretroviral presentaron un riesgo significativamente menor de mostrar lesiones orales OR: 0,32; 95\%, IC: 0,22$0,47$ ( $p=0,005)(20)$; dichas diferencias se podrían explicar ya que el número de la muestra de Tappuni AR et al y Hamza OJ et al, discrepa con la de este estudio; también que presentaron más alternativas de tratamiento farmacológico como combinaciones con tres fármacos y el número de pacientes incluidos en el grupo de monoterapia del presente estudio estaban en desproporción comparándolo con el grupo de biterapia, además en la fundación número 2 tenían un odontólogo dentro de la institución lo cual mejora las condiciones de salud oral ya que la asistencia odontológica es pronta y continua, algo que 
no sucedió con los pacientes de la fundación número 1 los cuales eran remitidos a las respectivas empresas prestadoras de servicio donde estaban inscritos siguiendo una serie de requisitos que postergaba la atención odontológica; por lo que es importante realizar mayor seguimiento a pacientes con monoterapia para visualizar realmente la presencia de lesiones en esta cohorte.

En relación con los niveles de linfocitos CD4 el grupo de pacientes que más manifestaciones orales presentó fue el de valores menores a $200 \mathrm{cell} / \mathrm{mm}^{3}$ $40,3 \%$, mientras que Tappuni AR et al, encontraron diferencias significativas con relación a los CD4/ $\mathrm{mm}^{3}$ pero igualmente fue el grupo que más manifestaciones reportó (13), en el estudio de NicolatouGalitis $\mathrm{O}$ et al, teniendo en cuenta sus resultados afirmaron que las lesiones orales se incrementaron significativamente en los pacientes con recuento de CD4 menor 200 cell $/ \mathrm{mm}^{3} \mathrm{p}<0,001$ (21), Bravo IM et al, en su estudio mostraron que los pacientes con niveles de CD4 menores a $200 \mathrm{cell} / / \mathrm{mm}^{3}$, fueron más susceptibles a desarrollar lesiones asociadas a VIH (17); este resultado está ampliamente relacionado con la inmunosupresión, entre más comprometido inmunológicamente se encuentre el paciente más manifestaciones va a presentar. Kolokotronis A et al, en su estudio reportan la candidiasis como manifestación oral más frecuente y está fuertemente asociada a cifras menores de 200 $\mathrm{cell} / \mathrm{mm}^{3} \mathrm{p}<0,02$ (22). Sin embargo en este estudio la candidiasis pseudomembranosa como manifestación oral asociada a VIH tiene menos probabilidades de presentarse cuando se tiene niveles de CD4 superiores a $500 \mathrm{cel} . / \mathrm{mm}^{3}$ mostrando valores estadísticamente significativos, con un OR: 0.297, IC: 0,103-0,852 ( $p<0,03)$.

Con relación a la carga viral donde mayormente se presentaron manifestaciones orales asociadas a $\mathrm{VIH}$, fueron en valores menores a 50.000 copias representando el 65,4\% donde ninguna de las patologías mostró asociación, Mellors et al y Baqui et al afirmaron que la progresión de la enfermedad es medida lo más exactamente posible por la carga viral creciente y que también se presenta un aumento en el número de lesiones orales asociadas a VIH $(23,24)$, Bravo IM et al, en un estudio reportaron que los pacientes con una carga viral de 30.000 copias, presentaron más lesiones bucales relacionadas con $\mathrm{VIH}$ independientemente del conteo de células CD4 (17), NicolatouGalitis $\mathrm{O}$ et al, reportaron que las lesiones orales se incrementaron significativamente en los pacientes con la carga viral mayor de 20.000 copias $p<0,001$ (21), da Silva CA et al, con una prevalencia de $8,3 \%$ en pacientes con cargas virales superiores a 10.000 copias (25), Baqui et al, correlacionaron la presencia de lesiones orales y carga viral en pacientes con VIH y encontró una asociación perceptible entre cargas virales superiores a 10.000 copias y enfermedad periodontal avanzada (24), Tappuni AR et al, en un estudio reportaron una prevalencia en pacientes con cargas virales superiores a 3.000 copias (13), en 2001 se realizó un estudio donde en una cohorte mexicana de pacientes con $\mathrm{VIH} / \mathrm{SIDA}$, que no recibían tratamiento antirretroviral, reveló que la aparición de candidiasis oral y leucoplasia vellosa se presento en pacientes con reducción sostenida de células CD4 y con un fuerte aumento de copias en la carga viral (26); teniendo en cuenta lo reportado por todos los autores antes mencionados se podría decir que entre más se incremente el número de copias del virus existe mayor riesgo de presentar manifestaciones orales.

\section{CONCLUSIONES}

Los pacientes tratados con monoterapia como terapéutica farmacológica antirretroviral presentaron menos manifestaciones orales que aquellos tratados con biterapia existiendo menos riesgo de presentar manifestaciones orales si se administra un solo fármaco antirretroviral, la candidiasis como manifestación oral asociada a VIH está fuertemente relacionadas a cifras menores de $200 \mathrm{cell} / \mathrm{mm}^{3}$ y que el subtipo pseudomembranosa tiene menos probabilidades de presentarse cuando se tiene niveles de CD4 superiores a $500 \mathrm{cel} . / \mathrm{mm}^{3}$

\section{BIBLIOGRAFÍA}

1. Pinzón E, Bravo S, Méndez F, Clavijo G, León M. Prevalence and factors related with the presence of oral manifestations in HIV/AIDS patients who attended health institutions in Cali, Colombia. Colomb Med 2008 Oct;39(4):346-55. 
2. UNAIDS/WHO. Situación de la epidemia de sida 2009. www.onusida.org.co.

3. Infección por VIH/SIDA en Colombia. Estado del arte 2005. Situación epidemiológica. www. onusida.org.co.

4. Gileva OS, Sazhina MV, Gileva ES, Efimov AV, Scully C. Spectrum of oral manifestationsof HIV/ AIDS in the Perm region (Russia) and identification of self-induced ulceronecrotic lingual lesions. Med Oral 2004 May;9(3):212-5.

5. Patton LL, Phelan JA, Ramos-Gomez FJ, Nittayananta W, Shiboski CH, Mbuguye TL. Prevalence and classification of HIV-associated oral lesions. Oral Dis 2002;8 Suppl 2:98-109.

6. Estrada J. Oral lesions associated to HIV infection and AIDS in individuals without antiretroviral treatment in Bogotá, DC. Biomédica 2001 Dec; 21(4):333-44.

7. Coogan M, Greenspan J, Challacombe SJ. Oral lesions in infection with human immunodeficiency virus. Bull World Health Organ 2005; 83(9):700-6.

8. Landerberger B, Egger M, Opravil M, et al. Clinical progression and virological failure on highly active antiretroviral therapy in HIV-1 patients: a prospective cohort study. Swiss HIV Cohort study. Lancet 1999 Mar;353(9156):863-8.

9. Powderly WG, Saag MS, Chapman S, et al. Predictors of optimal virological response to potent antiretroviral therapy. AIDS 1999 Oct;13 (14):1873-80

10. Moreno S, Arrizabalaga J, Gatell JM, Clotet B, Aguirrebengoa K, Antela A, et al. Recommendations on antiretroviral treatment. The AIDS Study Group of the Spanish Society of Infectious Diseases and Clinical Microbiology. Med Clin1998 Jan;110(3) 109-16.

11. Jané-Salas E, Chimenos-Küstner E, López-López J, Roselló-Llabrés X, Ocaña-Rivera I. Efecto de los tratamientos antirretrovirales en las mani- festaciones orales de los pacientes $\mathrm{VIH}+$. Av. Odontoestomatol 2006; 22(6):315-26.

12. Aquirre JM, Echebarria MA, Ocina E, Ribacoba L, Montejo M. Reduction of HIV-associated oral lesions after highly active antiretroviral therapy. Oral Surg Oral Med Oral Pathol Oral Radiol Endod 1999 Aug 88(2):114-5.

13. Tappuni AR y Fleming GJ. The effect of antiretroviral therapy on the prevalence of oral manifestations in HIV-infected patients: a UK study. Oral Surg Oral Med Oral Pathol Oral Radiol Endod 2001 Dec;92(6):623-8.

14. Rodríguez M. Manifestaciones orales asociadas con la infección por VIH-SIDA. Rev Cubana Estomatol 2005 Ene 42;(1).

15. Ranganathan K, Umadevi M, Saraswathi TR, Kumarasamy N, Solomon S, Johnson N. Oral lesions and conditions associated with human immunodeficiency virus infection in 1000 South Indian patients. Ann Acad Med Singapore 2004 Jul;33(4):37-42.

16. Lourenço AG, Figueiredo LT. Oral lesions in HIV infected individuals from Ribeirão Preto, Brazil. Med Oral Patol Oral Cir Bucal. 2008 May 1;13 (5):281-6.

17. Bravo IM, Correnti M, Escalona L, Perrone M, Brito A, Tovar V, Rivera $\mathrm{H}$. Prevalence of oral lesions in HIV patients related to CD4 cell count and viral load in a Venezuelan population. Med Oral Patol Oral Cir Bucal. 2006 Jan 1;11(1): 33-9.

18. Miziara ID, Weber R. Oral lesions as predictors of highly active antiretroviral therapy failure in Brazilian HIV-infected children. J Oral Pathol Med 2008 Feb;37(2):99-106.

19. Ceballos-Salobreña A, Gaitaín-Cepeda L, Ceballos-García L, et al. Prevalence of oral lesions by Candida sp: Their varieties and serotypes in a population of patients with AIDS under a highly active antiretroviral therapy. Rev Iberoam Micol 1998 Jul;15:141-5. 
20. Hamza OJ, Matee MI, Simon EN, Kikwilu E, Moshi MJ, Mugusi F, Mikx FH, Verweij PE, van der Ven AJ. Oral manifestations of HIV infection in children and adults receiving highly active antiretroviral therapy [HAART] in Dar es Salaam, Tanzania. BMC Oral Health 2006 Aug 18;6:12.

21. Nicolatou-Galitis O, Velegraki A, Paikos S, Economopoulou P, Stefaniotis T, Papanikolaou IS, Kordossis T. Effect of PI-HAART on the prevalence of oral lesions in HIV-1 infected patients. A Greek study. Oral Dis 2004 May;10 (3):145-50.

22. Kolokotronis A, Kioses V, Antoniades D, Mandraveli K, Doutsos I, Papanayotou P. Immunologic status in patients infected with HIV with oral candidiasis and hairy leukoplakia. Oral Surg Oral Med Oral Pathol 1994 Jul;78(1): 41-6.

23. Mellors JW, Rinaldo CR Jr, Gupta P, White RM, Todd JA, Kingsley LA. Prognosis in HIV-1 infection predicted by the quantity of virus in plasma. Science 1996 May 24;272(5265):1167-70.
24. Baqui A, Meiller T, Jabra-Rizk M, Zhang M, Kelley J, Falkler W. Association of HIV viral load with oral diseases. Oral Dis 1999 Oct;5(4):294-8.

25. Da Silva CA, Dourado I, Dahia SR, Harzheim E, Rutherford GW. Oral manifestations of HIV infection in patients receiving highly active antiretroviral therapy (HAART) in Bahia, Brazil. J Public Health Dent 2008 Summer;68(3):178-81.

26. Blignaut E, Patton LL, Nittayananta W, RamirezAmador V, Ranganathan K, Chattopadhyay A. (A3) HIV Phenotypes, oral lesions, and management of HIV-related disease. Adv Dent Res 2006 Apr 1;19(1):122-9.

\section{CORRESPONDENCIA}

Jonathan Harris Ricardo

Facultad de Odontología Universidad de Cartagena. Barrio Zaragocilla Campus de la Salud. Cartagena. D.T y C. Colombia. Correos electrónicos: j.harris.r@hotmail.com mcjimenezm@gmail.com 